\title{
Glycolysis module activated by hypoxia-inducible factor $1 \alpha$ is related to the aggressive phenotype of hepatocellular carcinoma
}

\author{
TAKASHI HAMAGUCHI ${ }^{1}$, NORIO IIZUKA ${ }^{1,2}$, RYOUICHI TSUNEDOMI ${ }^{1}$, YOSHIHIKO HAMAMOTO ${ }^{3}$, \\ TAKANOBU MIYAMOTO ${ }^{3}$, MICHIHISA IIDA ${ }^{1}$, YOSHIHIRO TOKUHISA ${ }^{1}$, KAZUHIKO SAKAMOTO ${ }^{1}$, \\ MOTONARI TAKASHIMA ${ }^{1}$, TAKAO TAMESA ${ }^{1}$ and MASAAKI OKA ${ }^{1}$ \\ Departments of ${ }^{1}$ Digestive Surgery and ${ }^{2}$ Complementary Medicine of Applied Molecular Bioscience, \\ Yamaguchi University Graduate School of Medicine, 1-1-1 Minami-Kogushi, Ube, Yamaguchi 755-8505; \\ ${ }^{3}$ Department of Biomolecular Engineering of Applied Molecular Bioscience, Yamaguchi University \\ Graduate School of Medicine 2-16-1 Tokiwadai, Ube, Yamaguchi 755-8611, Japan
}

Received April 7, 2008; Accepted June 5, 2008

DOI: 10.3892/ijo_00000058

\begin{abstract}
An increased level of glycolysis, an intracellular hallmark of neoplasms, enables cancer cells to survive under various conditions. To elucidate the role of increased glycolysis in the progression of hepatocellular carcinoma (HCC), we investigated the associations between the expression patterns of 14 glycolysis-related genes and clinicopathologic factors in 60 HCCs by using pooled transcriptome data. We then evaluated the therapeutic efficacy of the knockdown of ENO1, which is encoded by a glycolysis-related gene, in HCC cells. Among the 14 genes, levels of 8 genes (GPI, ALDOA, TPI1, GAPD, PGK, PGAM, ENOI and PKM), all of which can be transcriptionally activated by hypoxiainducible factor $1 \alpha(\mathrm{HIF}-1 \alpha)$, were significantly higher in HCC with venous invasion (VI) than in HCC without VI. Our cluster analysis showed that HCC patients with activation of the $8 \mathrm{HIF}-1 \alpha$-regulated genes had significantly shorter overall survival $(\mathrm{P}=0.023)$ than did HCC patients without increased expression levels of these genes. The association between the levels of ENOI and VI was confirmed in an independent sample set of 49 HCCs by real-time reversetranscription PCR. The knockdown of ENO1 by small-interfering RNA significantly inhibited the proliferation of an HCC cell line (HLE cells) in both the glucose-rich and glucosefree conditions, accompanied by a decreased $\mathrm{S}$ phase and increased G2/M phase of the cell cycle. Collectively, these
\end{abstract}

Correspondence to: Dr Masaaki Oka, Department of Surgery II, Yamaguchi University Graduate School of Medicine, 1-1-1 MinamiKogushi, Ube, Yamaguchi 755-8505, Japan

E-mail: 2geka-1@po.cc.yamaguchi-u.ac.jp

Key words: HCC, microarray, module, enolase, glycolysis data suggest that activation of an HIF-1 $\alpha$-regulated glycolysis module is closely related to the aggressive phenotype of $\mathrm{HCC}$, and that ENO1, a glycolysis module gene, might serve as a new target to circumvent HCC metastasis.

\section{Introduction}

An increased level of glycolysis in the energy production system of cancer cells was initially found by the renowned biochemist Otto Warburg (1). Since his discovery, many studies have revealed the molecular basis by which glycolysis is increased in cancer cells, particularly the regulation system of glycolysis-related enzymes, such as the hypoxic condition, oncogenic signals, and the Myc oncoprotein (2,3). As an example of the application of the Warburg effect to daily clinical practice, we can detect a cancerous nodule as an area where increased glucose uptake is visualized by positron emission tomography (PET) (4). However, applications of the Warburg effect to cancer therapy have not yet been found because of a lack of studies to comprehensively analyze glycolysis module genes or their encoded proteins in cancer progression.

Hepatocellular carcinoma (HCC) is the fourth most common malignancy in Japan (5) and the fifth most common malignancy worldwide (6). Despite advances in the conventional clinical treatment of $\mathrm{HCC}$, the prognosis remains poor because of the high recurrence rate after resection (7). To clarify the molecular basis underlying the metastasis of $\mathrm{HCC}$, we have performed genome-wide studies (8-10) in association with an aggressive phenotype of HCC. In the present study, we searched for glycolysis module genes among thousands of genes in the pooled data (8-10), and we investigated the relationship between the selected module genes and the clinical behavior of HCC. We discovered a new molecular subclass of HCC with poor prognosis; this subclass is characterized by the induction of several glycolysis module genes. Additionally, we show for the first time the therapeutic efficacy of $\alpha$-enolase (ENO1), one of the glycolytic enzymes, on HCC cells by using small-interfering RNA. 
Table I. Summary of glycolysis module genes and aggressive phenotype of HCC.

\begin{tabular}{|c|c|c|c|c|c|c|c|c|c|}
\hline $\begin{array}{l}\text { Glycolytic } \\
\text { pathway }\end{array}$ & Enzymes & Symbol $^{\mathrm{a}}$ & $\begin{array}{l}\text { Probe } \\
\text { number }\end{array}$ & Assessment & $\begin{array}{l}\text { HIF- } 1 \alpha \\
\text { regulation }\end{array}$ & $\begin{array}{l}\text { Tumor } \\
\text { size }\end{array}$ & $\begin{array}{c}\text { Tumor } \\
\text { differentiation }\end{array}$ & $\begin{array}{l}\text { Venous } \\
\text { invasion }\end{array}$ & Stage $^{b}$ \\
\hline \multicolumn{10}{|l|}{ Glucose } \\
\hline$\downarrow$ & HK & $H K 1$ & M75126_at & Eligible & Yes & N.S. & N.S. & N.S. & N.S. \\
\hline$\downarrow$ & & $H K 2$ & Z46376_rna1_at & Ineligible & Yes & & & & \\
\hline$\downarrow$ & & $H K 3$ & U51333_s_at & Eligible & & N.S. & N.S. & N.S. & N.S. \\
\hline$\downarrow$ & & $H K 4$ & M90299_at & Eligible & & N.S. & N.S. & N.S. & N.S. \\
\hline \multicolumn{10}{|c|}{ Glucose-6-phosphate } \\
\hline$\downarrow$ & GPI & GPII & K03515_at & Eligible & Yes & N.S. & N.S & $\mathrm{P}=0.001$ & N.S. \\
\hline \multicolumn{10}{|c|}{ Fructose-6-phosphate } \\
\hline$\downarrow$ & PFK & $P F K L$ & X15573_at & Ineligible & & & & & \\
\hline$\downarrow$ & & $P F K M$ & U24183_s_at & Ineligible & & & & & \\
\hline$\downarrow$ & & PFKP & D25328_at & Ineligible & & & & & \\
\hline \multicolumn{10}{|c|}{ Fructose-1,6-bisphosphate } \\
\hline$\downarrow$ & ALDO & $A L D O A$ & X12447_at & Eligible & Yes & N.S. & N.S. & $\mathrm{P}<0.001$ & $\mathrm{P}=0.022$ \\
\hline$\downarrow$ & & $A L D O B$ & M15656_at & Eligible & & N.S. & N.S. & N.S. & N.S. \\
\hline$\downarrow$ & & $A L D O C$ & X05196_at & Eligible & & N.S. & N.S. & N.S. & N.S. \\
\hline \multicolumn{10}{|c|}{ Dihydroxyacetone phosphate } \\
\hline$\downarrow$ & TPI & $T P I 1$ & HG2279 & Eligible & Yes & N.S. & N.S. & $\mathrm{P}=0.014$ & $\mathrm{P}=0.033$ \\
\hline \multicolumn{10}{|c|}{ Glyceraldehyde-3-phosphate } \\
\hline$\downarrow$ & GAPD & $G A P D$ & X01677_f_at & Eligible & Yes & N.S. & N.S. & $\mathrm{P}=0.014$ & N.S. \\
\hline \multicolumn{10}{|c|}{ 1,3-Bisphosphoglycerate } \\
\hline$\downarrow$ & PGK & $P G K$ & V00572_at & Eligible & Yes & N.S. & N.S. & $\mathrm{P}=0.007$ & N.S. \\
\hline$\downarrow$ & & $P G K 2$ & X05246_at & Ineligible & & & & & \\
\hline \multicolumn{10}{|c|}{ 3-Phosphoglycerate } \\
\hline$\downarrow$ & PGAM & $P G A M-B$ & J04173_at & Eligible & Yes & N.S. & N.S. & $\mathrm{P}=0.005$ & N.S \\
\hline$\downarrow$ & & $P G A M-M$ & J05073_at & Ineligible & & & & & \\
\hline \multicolumn{10}{|c|}{ 2-Phosphoglycerate } \\
\hline$\downarrow$ & ENO & ENO1 & M14328_s_at & Eligible & Yes & N.S. & N.S. & $\mathrm{P}=0.002$ & $\mathrm{P}=0.01$ \\
\hline$\downarrow$ & & ENO2 & X51956_rna1_at & Ineligible & & & & & \\
\hline$\downarrow$ & & ENO3 & X16504_s_at & Ineligible & & & & & \\
\hline \multicolumn{10}{|c|}{ Phosphoenolpyruvate } \\
\hline$\downarrow$ & PK & $P K M$ & X56494_at & Eligible & Yes & N.S. & $\mathrm{P}<0.001$ & $\mathrm{P}=0.02$ & N.S. \\
\hline$\downarrow$ & & $P K L R$ & M15465_s_at & Eligible & & N.S. & N.S. & N.S. & N.S. \\
\hline Pyruvate & & & & & & & & & \\
\hline
\end{tabular}

N.S., not significant. HIF-1 $\alpha$ hypoxia-inducible factor $1 \alpha .{ }^{a}$ Gene abbreviations are used based on Entrez Pubmed (http//www.ncbi.nlm.nih.gov/sites/entrez/). ${ }^{b}$ Stage based on the UICC TNM classification.

\section{Materials and methods}

Patients and pooled transcriptome data. By using high-density oligonucleotide arrays (HuGeneFL Array, Affymetrix, Santa Clara, CA), we previously analyzed the expression levels of 7,070 genes in $60 \mathrm{HCC}$ tissues from $60 \mathrm{HCC}$ patients who underwent curative hepatectomy and then received follow-up care $(8,9)$. The pooled data and the clinicopathologic features of the $60 \mathrm{HCCs}$ are listed on our web site (http://surgery2. med.yamaguchi-u.ac.jp/research/DNAchip/hcc-recurrence/ index.html) according to the Minimum Information About a Microarray Experiment (MIAME) (11) and TNM classification of the Union Internationale Contre le Cancer (UICC) (12). Written informed consent was obtained from all patients before surgery. The study protocol was approved by the Institutional Review Board for Human Use at the Yamaguchi University School of Medicine. All patients were followed up at least once every 3 months after surgery by ultrasonography (US), computed tomography (CT) scan, or magnetic resonance imaging (MRI), in addition to the measurement of 
Table II. ENO1 mRNA levels in newly enrolled 49 HCC samples.

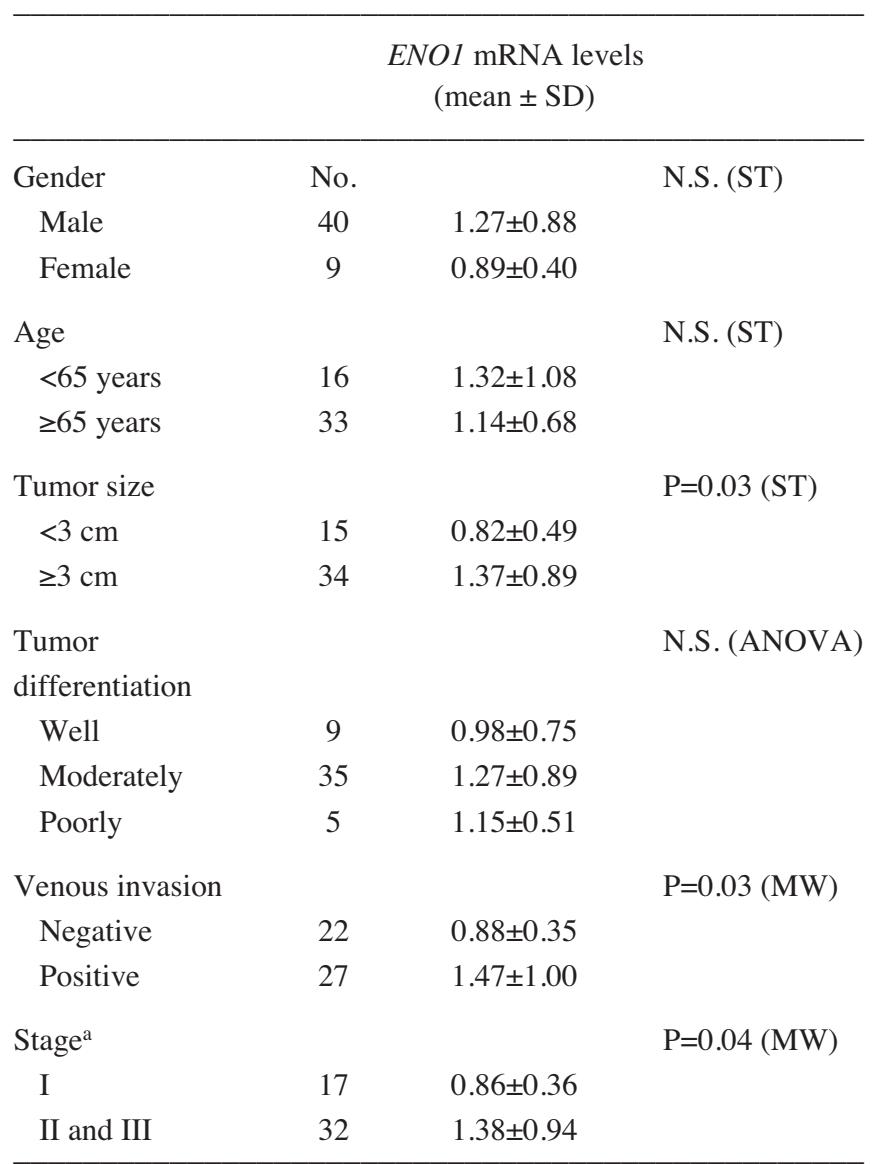

N.S., not significant. ST, Student's t-test. Anova, analysis of variance. MW, Mann-Whitney's U test. aStage based ov the UICC TNM classification.

serum $\alpha$-fetoprotein and protein induced by vitamin $\mathrm{K}$ absence II (PIVKA-II), as described previously (13). When tumor recurrence was suspected, hepatic angiography was included in the follow-up examination.

Selection of glycolysis-related genes. By using gene symbol (http://www.ncbi.nlm.nih.gov/sites/entrez/), we searched for genes whose products are involved in a series of reactions that metabolize glucose to pyruvate. As a result, we successfully extracted the raw data for 22 glycolysis-related genes from the data for 7,070 genes (Table I). Among the 22 genes, we omitted 8 genes whose levels were $<20$ in $>15 \%$ of the HCC cases. The remaining 14 genes were statistically assessed for associations with an aggressive phenotype of HCC.

Quantitation of ENO1 mRNA by real-time reverse-transcription $(R T)-P C R$. RNA isolation from both tissues and cell lines was performed with TRIzol reagent according to the manufacturer's instructions (Invitrogen Corp., Tokyo, Japan). First-strand cDNA was synthesized from the total RNA with the ExScript RT Reagent kit (Takara, Otsu, Japan). cDNA template (10 ng) was used for real-time PCR. A Light CyclerSystem and LightCycler FastStart DNA Master SYBR-Green
I (Roche Diagnostics, Tokyo, Japan) were used for real-time PCR. Expression levels of ENO1 mRNA were calculated as the ratio relative to $\beta$-actin (internal control), as described previously (14). The primers were 5'-GAGCTCCGGGAC AATGATAA and 5'-CTGTTCCATCCATCTCGATC for ENO1 and 5'-AACTCCATCATGAAGTGTGACG and 5-GATCCACATCTGCTGGAAGG for the $\beta$-actin gene.

Validation group. To validate the performance of ENO1, we used real-time RT-PCR to analyze ENO1 mRNA levels in 49 HCC tissues from 49 newly enrolled patients (Table II).

Cell culture. HLE cells, an HCC cell line, were cultured in Dulbecco's modified Eagle's medium (DMEM) (Nissui Pharmaceutical, Tokyo, Japan) containing 10\% heat-inactivated fetal bovine serum (FBS) (Invitrogen) supplemented with penicillin (100 units/ml), streptomycin (100 Ag/ml), and sodium bicarbonate $(1.5 \mathrm{~g} / \mathrm{l})$ at $37^{\circ} \mathrm{C}$ in $5 \% \mathrm{CO}_{2}$ in air. To examine the effect of the inhibition of glucose metabolism on cell proliferation, we used glucose-free DMEM (Invitrogen) containing 10\% dialyzed FBS (Invitrogen) instead of DMEM containing $10 \%$ FBS. The latter culture medium contains $<5 \mathrm{mg} / \mathrm{l}$ of D-glucose.

Knockdown of ENO1 protein. Endogenous ENO1 knockdown was performed by transfection of small-interfering RNA (siRNA) (sequence: 5'-AGCUGGUGCCGUUGAGAAG) specific for ENO1 mRNA into HLE cells. Lipofectamine 2000 (Invitrogen Corp.) was used for siRNA transfection. Trypsinized HLE cells were resuspended in Opti-MEM I (Promega, Tokyo, Japan) containing 5\% FBS. A complex of siRNA with Lipofectamine 2000 was administered to the suspended HLE cells. Twenty-four hours after transfection, the medium was replaced with glucose-containing or glucosefree DMEM, as mentioned above. A siRNA sequence (B-Bridge International Inc., Mountain View, CA) that does not match any human gene sequence was used as a negative control for ENO1 siRNA.

Western blot analysis. Western blot analysis was performed as described previously $(14,15)$. Anti-enolase polyclonal antibody (Santa Cruz Biotechnology, Inc., Santa Cruz, CA) and anti- $\beta$-actin polyclonal antibody (Sigma-Aldrich Co.) were used as the primary antibodies. Antigen detection was performed by using the ECL chemiluminescent assay (Amersham Biosciences, Tokyo, Japan).

Cell proliferation and cell cycle assays. Cell proliferation was determined by counting the number of viable cells. To determine the viability of cells, HLE cells were stained with Hoechst 33342 and propidium iodide (PI). Stained cells were counted under a fluorescence microscope (model IX71: Olympus Corp., Tokyo, Japan). HLE cells were harvested and fixed in cold $70 \%$ ethanol. Fixed cells were stained with PI. The cell-cycle stage was determined by measuring DNA content with flow cytometry (Epics XL, Beckman Coulter, Fullerton, CA).

Statistical analysis. Data for clinicopathologic characteristics were analyzed by the Student's t-test, Mann-Whitney U test, 
(A)

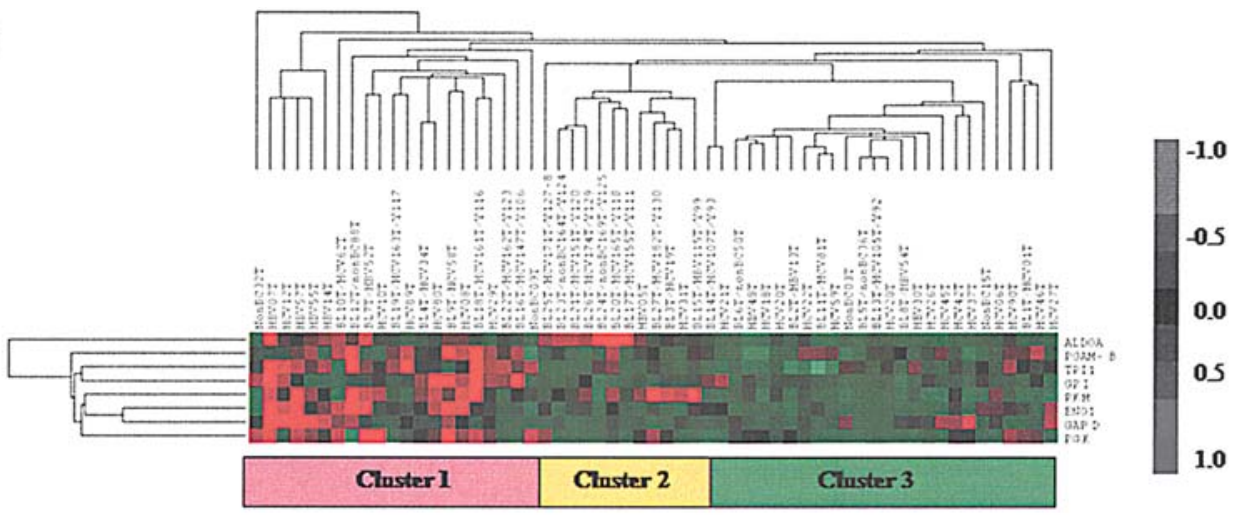

(B)

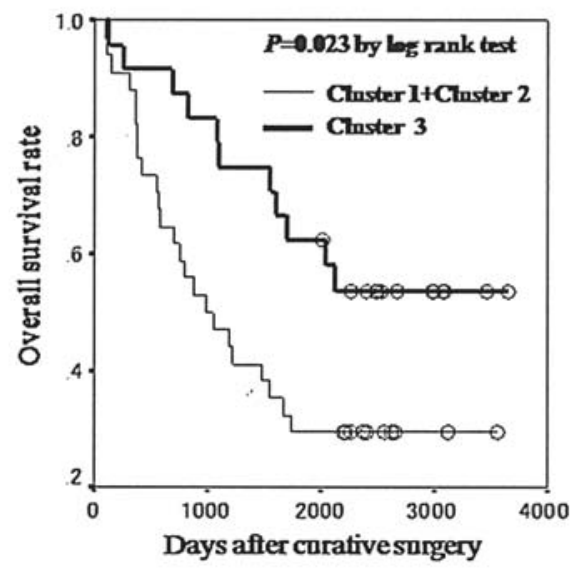

Figure 1. New molecular subclasses of HCC based on the expression patterns of 8 glycolysis module genes activated by HIF- $\alpha$. (A) Color displays of expression of 8 glycolysis module genes in $60 \mathrm{HCC}$ samples (http://surgery2.med.yamaguchi-u.ac.jp/research/DNAchip/) created with Cluster software and Tree View software (16). There are 3 major clusters according to the 8 gene expression levels. HCC in cluster 1 shows activation patterns of the 8 HIF- $\alpha$-regulated genes, HCC in cluster 3 shows their inactivation patterns, and HCC in cluster 2 shows intermediate patterns of their expression levels. (B) The relationship between the new molecular subclasses and patient outcome. HCC patients in clusters 1 and 2 had significantly shorter overall survival than did HCC patients in cluster 3 .

analysis of variance (ANOVA), and the log-lank test by using SPSS 11.0J software (SPSS, Inc., Chicago, IL). P<0.05 was accepted as statistically significant. A cluster analysis with 8 genes in 60 patients was performed according to the method of Eisen et al (16).

\section{Results}

We identified 22 glycolysis-related genes among the 7,070 genes on the DNA array platform (8). Fourteen of the 22 genes were judged to be eligible because of their abundant expression level (Table I). PKM gene expression was significantly higher in less-differentiated tumors than in welldifferentiated tumors $(\mathrm{P}<0.0001$ by ANOVA). The expression levels of 3 genes (ALDOA, TPI1, and ENO1) were significantly higher in advanced HCC (stages II and III) than in stage I HCC (Table I). We found that 10 of the 14 eligible glycolysis-related genes were transcriptional targets of hypoxia-inducible factor $1 \alpha(\mathrm{HIF}-1 \alpha)$ (3). Except for HK1 and HK2, levels of the remaining $8 \mathrm{HIF}-1 \alpha$-regulated genes (GPI, ALDOA, TPII, GAPD, PGK, PGAM, ENOI and $P K M$ ) were significantly higher in $\mathrm{HCC}$ with venous invasion (VI) than in HCC without VI (Table I).

Because all of the 8 downstream genes of HIF-1 $\alpha$ were related to VI of HCC, we used a cluster analysis (16) to define a new subclass of HCC with induction of HIF-1 $\alpha$-regulated glycolysis module genes. Our cluster analysis classified the 60 HCC patients into 3 clusters (Fig. 1A). HCC in cluster 1 showed activation of the $8 \mathrm{HIF}-1 \alpha$-regulated genes, HCC in cluster 3 showed their inactivation, and $\mathrm{HCC}$ in cluster 2 showed an intermediate pattern of expressions. HCC patients in clusters 1 and 2 had a significantly shorter overall survival than did HCC patients in cluster 3 ( $\mathrm{P}=0.023$ by log-rank test, Fig. 1B). The median disease-free survival periods of HCC patients in clusters 1 and 2 (combined) and cluster 3 were 419 days and 924 days, respectively; however, there was no statistically significant difference between the disease-free survival periods of the 2 groups (data not shown).

To confirm the reproducibility of our microarray data, we used real-time RT-PCR to investigate the ENO1 mRNA levels in an independent group of 49 HCC samples. We found that ENOI mRNA levels were significantly higher in HCCs with a larger tumor size $(>3 \mathrm{~cm}), \mathrm{HCCs}$ with VI, and HCCs of stages II and III than in those with smaller tumor size $(<3 \mathrm{~cm})$, those without VI, and those of stage I $(\mathrm{P}=0.03$, $\mathrm{P}=0.03$ and $\mathrm{P}=0.04$, respectively, Table II).

Transfection of a small-interfering RNA (siRNA), which was designed to inhibit the transcription of ENO1, into HLE cells markedly decreased both the mRNA and protein levels of ENO1 (Fig. 2A and B). The knockdown of ENO1 significantly inhibited the proliferation of HLE cells in both the 
A

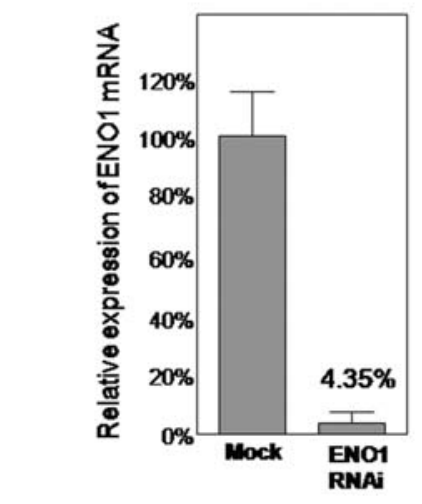

B

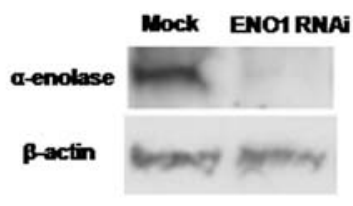

C
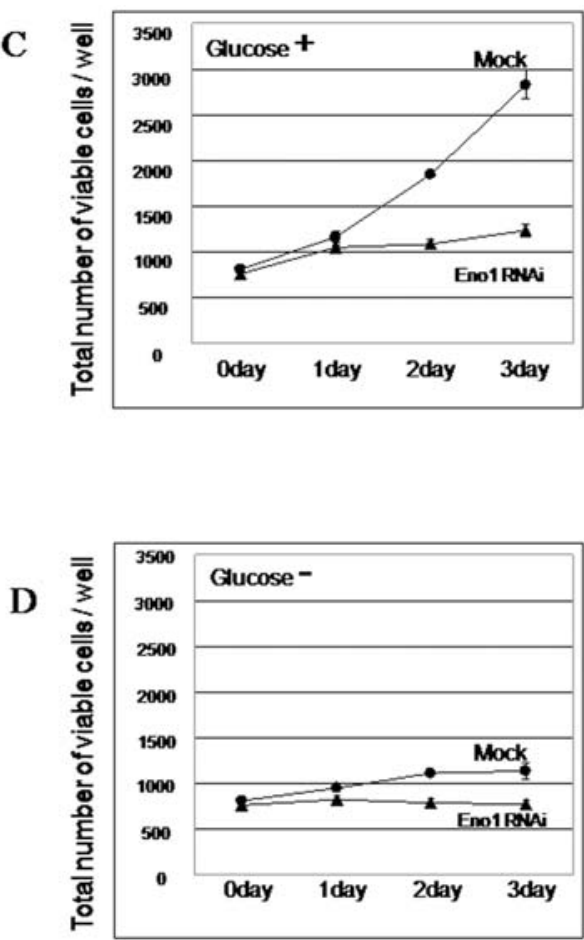

Figure 2. Inhibitory effect of ENO1 knockdown on the proliferation of HLE cells. (A) Assessment of the knockdown of the ENO1 gene by real-time RT PCR. Forty-eight hours after transfection of a small-interfering RNA (siRNA) targeting ENOI or a mock siRNA into HLE cells, total RNA was isolated from those cells. Levels of ENO1 mRNA were measured by real-time RT-PCR. Results are shown as the mean \pm SE of triplicate cultures. (B) Assessment of the knockdown of the ENO1 protein by Western blot. Cell lysates of ENO1 siRNA-transfected or control cells were prepared at $72 \mathrm{~h}$ after transfection. (C) Growth curves of ENO1 siRNAi-transfected cells and mock cells. Twenty-four hours after siRNA transfection, ENO1 siRNA-transfected and mock cells were cultured in glucose-containing medium (glucose ${ }^{+}$). The total number of viable cells was counted with the use of propidium iodide and Hoechst 33342 at each time indicated. Results are shown as the mean \pm SE of 5 cultures. (D) The same procedure was performed in glucose-free medium (glucose). Note that the knockdown of ENO1 significantly inhibited the proliferation of HLE cells in both the glucose-rich and glucose-free conditions (C and D).

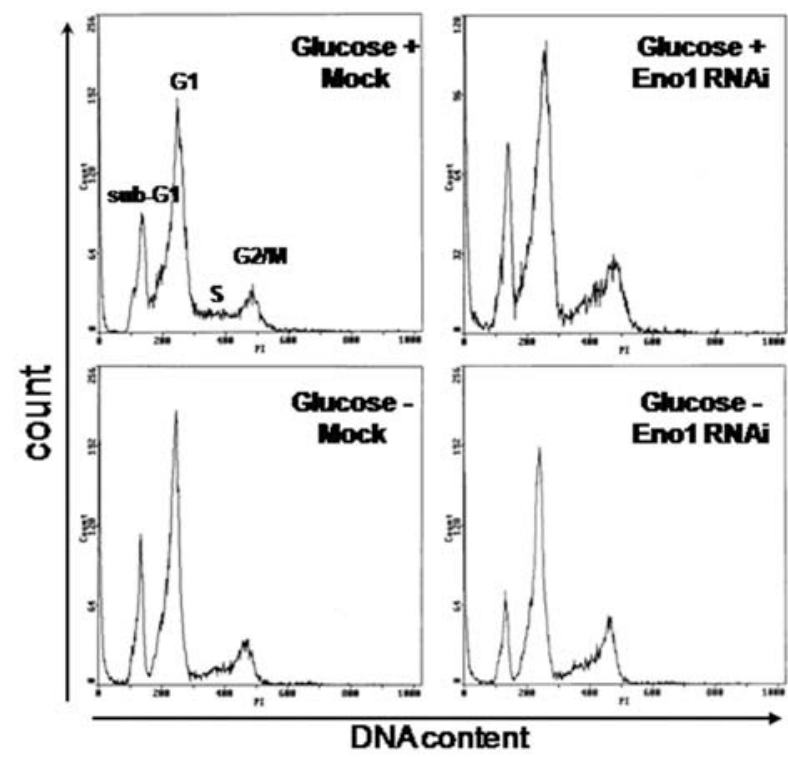

\begin{tabular}{|c|l|l|l|l|l|}
\hline & RNAi & \multicolumn{1}{|c|}{ sub G1 } & \multicolumn{1}{c|}{ G1 } & \multicolumn{1}{c|}{ S } & $\begin{array}{c}\text { G2M } \\
\text { (mean } \pm \text { S.E.) }\end{array}$ \\
\hline \multirow{2}{*}{+} & Mock & $20.4( \pm 0.69)$ & $47.37( \pm 0.41)$ & $9.89( \pm 0.16)$ & $9.33( \pm 0.46)$ \\
& ENO1 & $17.97( \pm 0.44)$ & $42.43( \pm 0.79)$ & $5.93( \pm 0.16)^{*}$ & $15.7( \pm 0.46)^{*}$ \\
\hline & Mock & $16.33( \pm 0.09)$ & $51.13( \pm 0.79)$ & $5.97( \pm 0.13)$ & $13.73( \pm 0.30)$ \\
& ENO1 & $12.17( \pm 0.20)$ & $47.57( \pm 0.61)$ & $5.92( \pm 0.06)$ & $2233( \pm 0.44)^{*}$ \\
\hline
\end{tabular}

Figure 3. Effect of ENO1 knockdown on the cell cycle of HLE cells. Cells were cultured in glucose-containing or glucose-free medium (glucose ${ }^{+}$or ${ }^{-}$) $24-72$ h after ENO1 or mock transfection. Subsequently, cells were harvested, fixed in cold $70 \%$ ethanol, and stained with propidium iodide. The cell cycle stage was determined by measuring DNA content with flow cytometry. ENO1 knockdown in HLE cells significantly decreased the S phase and increased the G2/M phase of the cell cycle in the glucose-rich condition, and it significantly increased the G2/M phase of the cell cycle in the glucose-free condition. 
glucose-rich and glucose-free conditions (Fig. 2C and D). The knockdown of ENO1 in HLE cells significantly decreased the $\mathrm{S}$ phase and increased the G2/M phase of the cell cycle in the glucose-rich condition, and the knockdown significantly increased the G2/M phase of the cell cycle in the glucosefree condition (Fig. 3).

\section{Discussion}

DNA microarray technology started a revolution in medical science upon its introduction in 1995 (17). This sophisticated technique has enabled a comprehensive genomic search to discover modules responsible for the metastatic potential of HCC (7). These modules include the immune response module $(8,9,18)$, detoxification module including $C Y P$ family genes (19), the cell adhesion module such as CD44, ITGA6, SPP1 (10) and Rho family genes $(20,21)$, the cell growth module such as TAF4B, SLC4A7, RAB38 and RYRI genes (22), the oncogenic module such as MET (23), and the ID2-related module (12,13). Additionally, our current study shows that a glycolysis module has a significant role in HCC metastasis.

Notably, levels of the 8 glycolysis module genes, all of which were transcriptionally activated by HIF-1 $\alpha$, were significantly higher in HCC with VI than in HCC without VI. Since VI is a hallmark of the intrahepatic spread of HCC cells and of poor outcome (24), we hypothesized that a molecular subclass of HCC determined by the 8-gene signature might be related to the outcome. Indeed, our cluster analysis showed that HCC patients with upregulation of the 8 glycolysis module genes had significantly shorter overall survival than did HCC patients without upregulation. It is known that HIF- $1 \alpha$ activation plays a key role in the maintenance of the glycolytic phenotype $(25,26)$, favors cancer invasion and metastasis via increased angiogenesis (27), and promotes cell proliferation (28). Thus, in conjunction with these reports and our present findings, it is reasonable to assume that $\mathrm{HIF}-1 \alpha$ can be involved in the metastatic potentials of HCC, and the downstream glycolysis module is a potent therapeutic target for preventing HCC metastasis.

The comprehensive analysis of cellular proteins is a central area of oncology research that is developing in the postgenome era $(29,30)$. Our previous proteomics study found that ENO1, one of the HIF-1 $\alpha$-regulated glycolytic enzymes, was overexpressed in HCC and that its level was associated positively with tumor progression (31). Many proteomics studies have reported increased levels of various glycolysisrelated enzymes in malignant tumors such as lung squamous cell carcinoma (32), pancreatic carcinoma (33), esophageal squamous cell carcinoma (34), HER-2/neu-positive breast carcinoma (35), and prostate carcinoma (36). More importantly, ENO1 is the only upregulated protein common to all of the cancer tissues in these studies (31-36). In the present study, we used an independent cohort of HCC patients to confirm that ENO1 mRNA levels were positively associated with VI of HCC. These findings prompted us to highlight the inhibitory effect of ENO1 knockdown on the proliferation of HCC cells. Our ENO1 knockdown strategy may have the advantage of fewer adverse effects, because we previously confirmed that the ENOI protein is overexpressed in HCC tissues compared with non-cancerous liver tissues (31). By contrast, the targeting of HIF-1 $\alpha$ would cause severe effects because of its broad range of biological function and its wide distribution in various organs $(27,28)$.

We also found that ENO1 knockdown significantly inhibited the proliferation of HLE cells even in glucosefree conditions. This finding suggests that ENO1 has other non-glycolytic functions, which may yield another tactic against $\mathrm{HCC}$ cells in addition to the metabolic advantage of the increased glycolysis in cancer cells. The ENO1 gene intrinsically encodes both $\alpha$-enolase and a shorter monomeric structural lens protein, tau-crystallin. $\alpha$-enolase is a full-length form that is found in the cytoplasm. The shorter tau-crystallin protein is produced from an alternative translation start of ENO1, and it is localized to the nucleus. This protein has been found to bind to an element in the c-myc promoter (37), and has been designated as the c-myc promoter-binding protein (MBP-1). Ghosh et al reported that knockdown of MBP-1 in prostate cancer cells perturbs cell proliferation by inhibiting cyclin A and cyclin B1 expression (37). This report supports our present finding of the nonglycolytic action of ENOI in cell proliferation. Thus, we must investigate whether the non-glycolytic action of ENO1 is specific for HCC cells or not. It also remains unclear why ENO1 knockdown allowed HLE cells to arrest in the G2/M phase of the cell cycle without an increase in apoptosis.

Our present study shows the significance of the HIF- $1 \alpha$ regulated glycolysis module in HCC progression by showing its predictive power for patient outcome and the therapeutic efficacy of ENO1 from among the HIF-1 $\alpha$-regulated glycolysis module genes. This HIF-1 $\alpha$-regulated glycolysis module could be important for the development of first-line treatments during the multiple metastatic cascades of HCC. Further studies are needed for deeper insights into our present findings and to develop a new therapy against HCC.

\section{Acknowledgements}

This work was supported by the Ministry of Education, Culture, Sports, Science and Technology (No. 18390366, No. 17591406 and Knowledge Cluster Initiative), the Venture Business Laboratory of Yamaguchi University and the New Energy and Industrial Technology Development Organization (Grant number: 03A02018a).

\section{References}

1. Warburg O: On the origin of cancer cells. Science 123: 309-314, 1956.

2. Kim JW and Dang CV: Cancer's molecular sweet tooth and the Warburg effect. Cancer Res 66: 8927-8930, 2006.

3. Shaw RJ: Glucose metabolism and cancer. Curr Opin Cell Biol 18: 598-608, 2006.

4. Gambhir SS: Molecular imaging of cancer with positron emission tomography. Nat Rev Cancer 2: 683-693, 2002.

5. Okita K: Clinical aspects of hepatocellular carcinoma in Japan. Intern Med 45: 229-233, 2006.

6. Llovet JM: Updated treatment approach to hepatocellular carcinoma. J Gastroenterol 40: 225-235, 2005.

7. Iizuka N, Hamamoto Y, Tsunedomi R and Oka M: Translational microarray systems for outcome prediction of hepatocellular carcinoma. Cancer Sci 99: 441-450, 2008.

8. Iizuka N, Oka M, Yamada-Okabe H, et al: Oligonucleotide microarray for prediction of early intrahepatic recurrence of hepatocellular carcinoma after curative resection. Lancet 361: 923-929, 2003. 
9. Matoba K, Iizuka N, Gondo T, et al: Tumor HLA-DR expression linked to early intrahepatic recurrence of hepatocellular carcinoma. Int J Cancer 115: 231-240, 2005.

10. Iizuka N, Tamesa T, Sakamoto K, Miyamoto T, Hamamoto Y and Oka M: Different molecular pathways determining extrahepatic and intrahepatic recurrences of hepatocellular carcinoma. Oncol Rep 16: 1137-1142, 2006.

11. Brazma A, Hingamp P, Quackenbush J, et al: Minimum information about a microarray experiment (MIAME)-toward standards for microarray data. Nat Genet 29: 365-371, 2001.

12. Sobin LH and Wittekind C: TNM Classification of Malignant Tumours. 6th edition. UICC, Wiley-Liss, pp81-83, 2002.

13. Tokuhisa Y, Iizuka N, Sakaida I, et al: Circulating cell-free DNA as a predictive marker for distant metastasis of hepatitis C virus-related hepatocellular carcinoma. Br J Cancer 97: 1399-1403, 2007.

14. Tsunedomi R, Iizuka N, Yamada-Okabe H, et al: Identification of ID2 associated with invasion of hepatitis $\mathrm{C}$ virus-related hepatocellular carcinoma by gene expression profile. Int J Oncol 29: 1445-1451, 2006.

15. Tsunedomi R, Iizuka N, Tamesa T, et al: Decreased ID2 promotes metastatic potentials of hepatocellular carcinoma by altering secretion of vascular endothelial growth factor. Clin Cancer Res 14: 1025-1031, 2008.

16. Eisen MB, Spellman PT, Brown PO and Botstein D: Cluster analysis and display of genome-wide expression patterns. Proc Natl Acad Sci USA 95: 14863-14868, 1998.

17. Schena M, Shalon D, Davis RW and Brown PO: Quantitative monitoring of gene expression patterns with a complementary DNA microarray. Science 270: 467-470, 1995

18. Uchimura S, Iizuka N, Tamesa T, Miyamoto T, Hamamoto Y and Oka M: Resampling based on geographic patterns of hepatitis virus infection reveals a common gene signature for early intrahepatic recurrence of hepatocellular carcinoma. Anticancer Res 27: 3323-3330, 2007.

19. Tsunedomi R, Iizuka N, Hamamoto Y, et al: Patterns of expression of cytochrome $\mathrm{P} 450$ genes in progression of hepatitis $\mathrm{C}$ virus-associated hepatocellular carcinoma. Int J Oncol 27: 661-667, 2005.

20. Okabe H, Satoh S, Kato T, et al: Genome-wide analysis of gene expression in human hepatocellular carcinomas using cDNA microarray: identification of genes involved in viral carcinogenesis and tumor progression. Cancer Res 61: 2129-2137, 2001.

21. Wang W, Yang LY, Huang GW, Lu WQ, Yang ZL, Yang JQ and Liu HL: Genomic analysis reveals RhoC as a potential marker in hepatocellular carcinoma with poor prognosis. Br J Cancer 90: 2349-2355, 2004.

22. Ho MC, Lin JJ, Chen CN, et al: A gene expression profile for vascular invasion can predict the recurrence after resection of hepatocellular carcinoma: a microarray approach. Ann Surg Oncol 13: $1474-1484,2006$.
23. Kaposi-Novak P, Lee JS, Gomez-Quiroz L, Coulouarn C, Factor VM and Thorgeirsson SS: Met-regulated expression signature defines a subset of human hepatocellular carcinomas with poor prognosis and aggressive phenotype. J Clin Invest 116: 1582-1595, 2006.

24. Vauthey JN, Lauwers GY, Esnaola NF, et al: Simplified staging for hepatocellular carcinoma. J Clin Oncol 20: 1527-1536, 2002.

25. Harris AL: Hypoxia: a key regulatory factor in tumour growth. Nat Rev Cancer 2: 38-47, 2002.

26. Semenza GL: Targeting HIF-1 for cancer therapy. Nat Rev Cancer 3: 721-732, 2003.

27. Gatenby RA and Gillies RJ: Why do cancers have high aerobic glycolysis? Nat Rev Cancer 4: 891-899, 2004.

28. Alberts B, Johnson A, Lewis J, Raff M, Roberts K and Walter P: Molecular biology of the cell. Garland Publishing, New York, 2002.

29. Petricoin EF, Zoon KC, Kohn EC, Barrett JC and Liotta LA: Clinical proteomics: translating benchside promise into bedside reality. Nat Rev Drug Discov 1: 683-695, 2002.

30. Kuramitsu Y and Nakamura K: Proteomic analysis of cancer tissues: shedding light on carcinogenesis and possible biomarkers. Proteomics 6: 5650-5661, 2006.

31. Takashima M, Kuramitsu Y, Yokoyama Y, et al: Overexpression of alpha enolase in hepatitis $\mathrm{C}$ virus-related hepatocellular carcinoma: association with tumor progression as determined by proteomic analysis. Proteomics 5: 1686-1692, 2005

32. Li C, Xiao Z, Chen Z, et al: Proteome analysis of human lung squamous carcinoma. Proteomics 6: 547-558, 2005.

33. Mikuriya K, Kuramitsu Y, Ryozawa S, et al: Expression of glycolytic enzymes is increased in pancreatic cancerous tissues as evidenced by proteomic profiling by two-dimensional electrophoresis and liquid chromatography-mass spectrometry/mass spectrometry. Int J Oncol 30: 849-855, 2007.

34. Qi Y, Chiu JF, Wang L, Kwong DL and He QY: Comparative proteomic analysis of esophageal squamous cell carcinoma. Proteomics 5: 2960-2971, 2005 .

35. Zhang D, Tai LK, Wong LL, Chiu LL, Sethi SK and Koay ES: Proteomic study reveals that proteins involved in metabolic and detoxification pathways are highly expressed in HER-2/neupositive breast cancer. Mol Cell Proteomics 4: 1686-1896, 2005.

36. Van den Bemd GJ, Krijgsveld J, Luider TM, van Rijswijk AL, Demmers JA and Jenster G: Mass spectrometric identification of human prostate cancer-derived proteins in serum of xenograftbearing mice. Mol Cell Proteomics 5: 1830-1839, 2006.

37. Ghosh AK, Steele R and Ray RB: Knockdown of MBP-1 in human prostate cancer cells delays cell cycle progression. J Biol Chem 281: 23652-23657, 2006. 